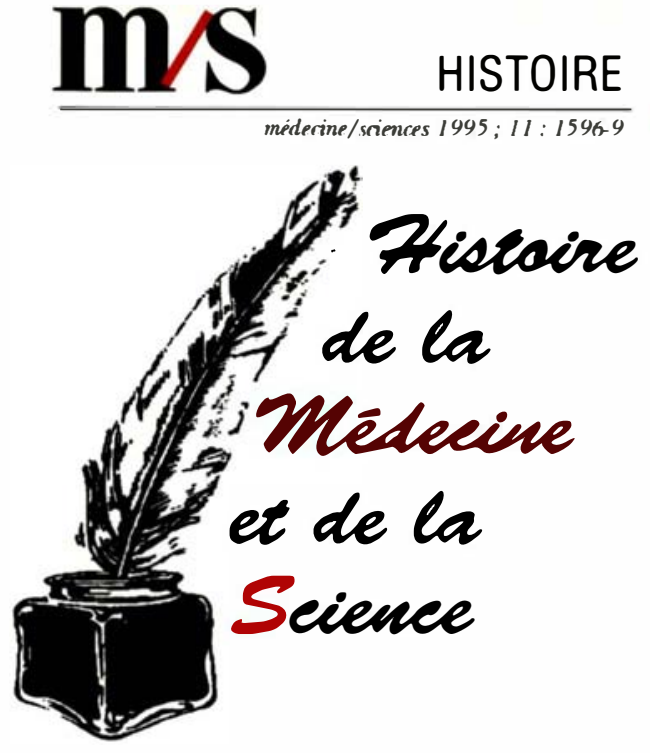

1896-1914 : une thérapie marginale

Si les applications médicales de la découverte de Roentgen sont presque immédiates (le premier ouvrage de radiologie paraît cinq mois après la publication de Roentgen), l'idée d'utiliser les rayons $\mathrm{X}$ à des fins thérapeutiques suit de très peu les premières expériences de radiologie. Les médecins découvrent très rapidement, à leurs dépens et à ceux des sujets soumis aux expérimentations, que si les rayons $\mathrm{X}$ donnent à voir des images de l'intérieur du corps, ils ont aussi la curieuse et douloureuse propriété de "brûler" la peau. Avec des effets parfois désastreux, la nouvelle arme thérapeutique sera utilisée dès 1896 [1].

S'ouvre alors une période de tâtonnements empiriques qui voit la radiothérapie expérimentée sur de très nombreuses affections (cancers, maladies cutanées, tuberculose, syphilis, épilepsie, maladies parasitaires, etc.). Les déboires sont nombreux, mais le facteur chance va jouer un rôle considérable. Malgré le fait que les médecins travaillent complètement à l'aveugle et avec des appareils extrêmement rudimentaires, certains cancers de la peau sont si sensibles aux rayons $\mathrm{X}$ qu'ils parviennent à être guéris. Les succès obtenus par Thor Stenbeck en 1899, confirmés par un autre Suédois, Tage Sjören, dans la cure d'un cancer cancroïde de la peau, amènent définitivement la radiothérapie dans l'arsenal médical antican-

\title{
Naissance et développement de la radiothérapie en France
}

Un certain nombre d'avancées cognitives et techniques permettent d'établir: (1) que les rayons $\mathrm{X}$ (et non l'électricité) sont bien l'agent responsable des effets biologiques observés après une exposition à un tube de Crookes en activité et que le radium produit des effets semblables ; (2) que les rayons $\mathrm{X}$ n'agissent pas par l'intermédiaire de la réaction inflammatoire qu'ils induisent, mais qu'ils exercent une action résolutive directe sur les cellules; (3) que cette action résolutive peut s'exercer sur les tissus des organes profonds et qu'une technique, la radiothérapie pénétrante, mise au point par l'Allemand Perthes, permet d'agir "en profondeur" en limitant les réactions inflammatoires au niveau de la peau; (4) que l'action biologique des rayons est élective : pour une même quantité de rayonnement absorbée, certains types de cellules sont détruits, d'autres "résistent". Ces conditions cognitives et techniques réunies, la question de l'action élective des rayons $\mathrm{X}$ sur les cellules peut être posée et trouver une première réponse cohérente et relationnelle sous forme d'une loi de corrélation entre la "fragilité roentgenienne des cellules et leur activité reproductrice ", établie en 1906 par Jean Bergonié et Albert Tribondeau, médecins français de Bordeaux. Cette «loi" permet de fonder la radiothérapie sur des bases scientifiques pertinentes [3].
Ces éléments brièvement rappelés, comment se construit le domaine de la radiothérapie en France jusqu'à la veille de la Première Guerre mondiale?

Le premier point qui mérite d'être mentionné tient au fait que les applications médicales de la découverte de Roentgen d'emblée "échappent ", si l'on peut dire, aux physiciens pour être prises en main par les médecins, au premier rang desquels les spécialistes "d'électricité médicale". Ceux-ci, à quelques exceptions notables, fourniront les premiers contingents de "radiologues " et, par voie de conséquence, c'est parmi eux que l'on trouvera la majeure partie des médecins s'investissant dans la radiothérapie. Cela n'est pas spécifique à la France. En revanche, ce qui l'est plus, c'est le statut de ces médecins électriciens par rapport aux élites hospitalo-universitaires. On peut, sans forcer le trait, dire que les médecins électriciens occupent une position dominée dans un champ médical où le pouvoir et le prestige sont concentrés dans les mains des "cliniciens ", c'est-à-dire des prof esseurs de clinique médicale ou chirurgicale. En France, la hiérarchie universitaire est très directement liée et subordonnée à la hiérarchie hospitalière et à ses concours. Or, le médecin électricien, s'il est professeur, est un universitaire titulaire d'une chaire de "science dite fondamentale ", il n'a pas suivi le cursus royal qui conduit de l'internat au médicat des hôpitaux; s'il travaille à 
l'hôpital, c'est dans un laboratoire et non dans un service ; et s'il traite des malades, c'est occasionnellement et sous la responsabilité d'un clinicien. Qui plus est, comme tout universitaire "fondamentaliste", il vit de son salaire de fonctionnaire, alors que l'élite clinicienne est une élite de médecins libéraux n'exerçant à l'hôpital qu'à mi-temps et gagnant sa vie grâce à sa clientèle privée... Il soigne, lui, l'élite sociale. Cét élément d'analyse est important à prendre en compte si l'on veut comprendre les déterminants historicostructurels qui pèsent sur la place de la radiothérapie dans l'univers hospitalier [4]. De fait, jusqu'à la guerre de 1914 , la radiothérapie restera une technologie de traitement pour l'essentiel confinée au laboratoire, entre les mains de non-cliniciens. En ce sens, on peut dire qu'elle demeure une thérapeutique expérimentale éprouvée, soit sur l'animal, soit sur des malades considérés comme des incurables. (e sont ces incurables que les chirurgiens consentiront en désespoir de cause - à adresser à leurs collègues radiologues.

Cie schéma où la chirurgie est la technique curative, et la radiothérapie la technique palliative, règne en maître partout dans le pays. Un domaine toutefois fait exception, la dermatologie. Pour cette spécialité médicale qui dépendait de la chirurgie pour le traitement des cancers de la peau, l'invention de la radiothérapie est une aubaine. L'efficacité des rayons $\mathrm{X}$ sur certaines formes de tumeurs malignes cutanées donne aux dermatologues la possibilité de se passer des chirurgiens. Autrement dit, là où la radiothérapie est utilisée comme traitement curatif, c'est la chirurgie qui est exclue. Contrairement à ce qui se passe à la même époque en Allemagne et dans tous les pays où des structures spécialisées dans le traitement du cancer voient le jour, l'association chirurgie-rayons $\mathrm{X}$ n'entre pas dans les pratiques hospitalières. Il faudra attendre les bouleversements liés à l'organisation de la médecine et de la chirurgie de guerre au cours du conflit de 1914-
1918 pour que les conditions d'une collaboration entre chirurgiens et radiothérapeutes soient réunies.

Avant la guerre, la radiothérapie est marginale. Elle a aussi comme caractéristique de ne pas être une pratique homogène. La conception que les dermatologues ont de la radiothérapie est celle d'un outil thérapeutique dont ils testent empiriquement l'efficacité sur les patients. Les médecins des laboratoires d'électroradiologie sont davantage portés à fonder l'usage des rayons $\mathrm{X}$ sur des bases rationnelles, en s'attachant notamment à comprendre les mécanismes de leur action biologique. Mais c'est de leur rencontre et de leur collaboration avec un troisième type de médecins que naîtront les avancées conceptuelles majeures de la radiothérapie. Cet autre groupe est réduit numériquement à quelques individualités ayant en commun d'être des histophysiologistes. Leur ambition est de faire évoluer l'histologie, science descriptive et morphologique, pour en faire une science expérimentale susceptible de devenir une des bases essentielles de la biologie. D'où l'importance des travaux sur la cytologie du testicule parce qu'ils mettent en évidence, en différenciant les cellules de la lignée germinale, un processus physiologique. C'est l'utilisation des rayons $\mathrm{X}$ qui va permettre de passer d'une histophysiologie descriptive du testicule à une histophysiologie expérimentale. Reprenant les expériences de radiologues qui avaient observé que l'irradiation de cobaye provoquait une azoospermie, l'histologiste Albert Tribondeau, associé au radiologue Alban Bergonié, démontre que les effets des rayons $\mathrm{X}$ sont maximaux sur les cellules souches de la lignée germinale, c'est-à-dire les cellules qui se divisent le plus, qui sont le moins différenciées, alors que les spermato\%oïdes, cellules différenciées, sont résistants. C'est de ce travail que va être tirée la " loi de corrélation entre la fragilité roentgenienne des cellules et leur activité reproductrice". Son retentissement amène d'autres histologistes, comme Dominici à Paris et Claudius Regaud à Lyon, à s'intéresser aux radiations comme instrument expérimental d'une utilité cruciale pour l'histophysiologie. Ainsi Dominici établit-il une loi de corrélation analogue à celle de Tribondeau pour l'action biologique du radium. Mais ces histologistes, pour mieux maîtriser l'instrument expérimental, s'investissent dans des recherches visant à préciser les modalités de son action et à perfectionner les techniques d'irradiation. Qui plus est, afin de vérifier les principes de la radiothérapie établis en laboratoire, ils se tournent vers la clinique et obtiennent des chirurgiens que leur soient confiés quelques malades incurables. Les traitements échouent, mais permettent à Regaud, par exemple, d'établir qu'un traitement par des doses successives mais trop espacées induit une décroissance de la radiosensibilité des tumeurs malignes. Autrement dit, l'expérimentation sur le malade, par les questions qu'elle soulève, permet d'enrichir la connaissance des propriétés biologiques des rayons $\mathrm{X}$. Mais, parce que le projet des histophysiologistes s'intéresse aux radiations comme "instrument expérimental ", ceux-ci lient études sur les rayons $\mathrm{X}$ et études sur le radium. ('est ainsi que lorsque, en 1913, l'Institut Pasteur et la faculté des sciences conjuguent leurs efforts pour mettre sur pied un Institut du radium à Paris, c'est à Claudius Regaud qu'ils font appel pour en diriger la section de recherche biologique. Son poste est couplé à celui de directeur d'un laboratoire d'histologie créé spécialement pour lui à l'Institut Pasteur. C'est au moment où celui-ci obtient enfin les conditions institutionnelles lui permettant de développer des recherches articulant histophysiologie et étude des radiations que la guerre éclate. Regaud part au front. Mais quand il reviendra quatre ans plus tard, il renoncera à son projet histologique pour s'investir dans la lutte contre le cancer [5].

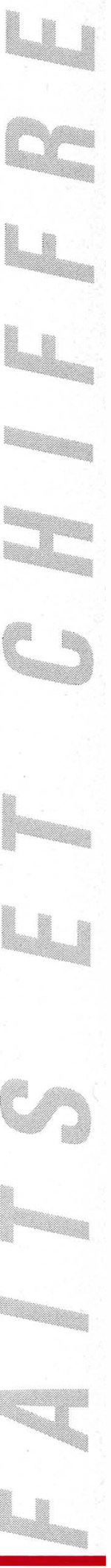


1920-1940 : la radiothérapie, modèle de la médecine d'avenir

Je ne peux ici qu'évoquer très schématiquement l'ensemble des bouleversements liés à la guerre, qui vont amener un changement en profondeur de la place de la radiothérapie. Disons simplement que c'est avec la guerre qu'émerge en France une nouvelle représentation faisant $\mathrm{du}$ cancer un fléau social au même titre que la tuberculose, la syphilis et l'alcoolisme, que cette représentation trouve sa formalisation institutionnelle avec la naissance de la Ligue contre le cancer, dont un des objectifs est de créer en France des centres de traitement spécialisés associant chirurgie, traitements par les radiations (rayons $\mathrm{X}$ et radium) et laboratoire d'anatomopathologie, et que ces centres sont pensés en fonction du modèle de collaboration pluridisciplinaire rendu nécessaire par la chirurgie de guerre. C'est aussi pendant la guerre que Claudius Regaud, devenu collaborateur de Justin Godart, le responsable du secrétariat d'État au service de santé des armées, fait l'apprentissage du pouvoir et accumule un capital considérable de relations sociales, tant avec l'élite hospitalo-universitaire qu'avec l'élite "mondaine" parisienne. C'est en tirant parti de ces relations qu'il parvient à transformer la section biologique de l'Institut du radium en centre anticancéreux expérimental lié à l'hôpital Pasteur : la Fondation Curie. Pensée par un chercheur expérimentaliste, la Fondation réalise un modèle institutionnel de "médecine scientifique" qui n'aura pas d'équivalent à l'époque en France. L'institution associe laboratoire de recherche fondamentale sur les radiations, laboratoire de recherche appliquée à l'instrumentation médicale des radiations et services cliniques conçus comme des lieux d'expérimentation thérapeutique. La thérapeutique à la Fondation Curie obéira à une logique d'innovation permanente, chaque année voyant naître de nouveaux par rapport aux hôpitaux de l'Assistance publique et à la Faculté de médecine, elle restera une institution unique en son genre, mais pèsera par ses réalisations sur l'évolution des autres centres anticancéreux [5]. Sous la pression de la Ligue contre le cancer, le gouvernement français se décide à impulser une politique anticancéreuse. Un réseau de centres spécialisés est construit en 1922 et 1930. Bien qu'encore très rudimentaires, les appareils de radiothérapie et de radiumthérapie atteignent des coûts financiers qui désorientent et effraient les responsables de la santé [6]. La question du nombre optimal de centres anticancéreux à créer est au centre des débats sur le cancer au Parlement en 1925. Deux positions s'affrontent. La première est défendue par certains députés provinciaux qui appuient les chirurgiens libéraux, chefs de service à l'hôpital de leur ville, désireux de voir se développer sous leur direction un centre. Leur conception des centres anticancéreux se rapproche du modèle de la lutte antituberculeuse. Il faut, disentils, construire un réseau de petits centres couvrant l'ensemble des villes grandes et moyennes. Cette vision recouvre une conception de la stratégie thérapeutique où la chirurgie domine et où la radiothérapie fait fonction de force d'appoint. Elle va de pair avec l'idée que les traitements par le radium ou les rayons $\mathrm{X}$ n'évolueront guère par rapport à l'état actuel des techniques. Un, deux ou trois au maximum appareils de radiothérapie pénétrante, quelques dizaines d'aiguilles de radium (soit quelques centaines de milligrammes du produit) sont dans cette optique suffisants pour équiper un centre.

La seconde conception, qui l'emporte, est défendue par le ministre de la Santé Justin Godart qui s'appuie sur un rapport écrit par son ami Claudius Regaud. La vision de Regaud est radicalement différente. Elle anticipe le développement de la cancérologie à l'image de ce qui se passe à la Fondation Curie. Pour lui, la radiothérapie est toujours dans un processus d'évolution lié à la marche des innovations thérapeutiques. Diviser les subsides de l'Etat et les fonds privés sur un grand nombre de centres n'aboutirait, en limitant les ressources de chaque centre, qu'à bloquer les progrès à venir. Car ces progrès ne peuvent venir que d'innovations technologiques augmentant la puissance d'irradiation des appareils. En 1925, la Fondation Curie est la seule institution à disposer d'une grande quantité de radium. Grâce aux appuis relationnels et au prestige dont jouit Marie Curie, la Fondation a attiré les dons et la Compagnie belge des mines de radium du Kitanga a mis à sa disposition plusieurs grammes du produit. Cette grande quantité de radium a rendu possible la réalisation d'une innovation technique majeure: la radiumthérapie à distance. Contrairement à la technique utilisée jusque-là qui mettait en jeu les propriétés d'irradiation de contact au radium (d'où l'emploi d'aiguilles contenant quelques milligrammes), la nouvelle technique est fondée sur l'irradiation à distance du radium. Mais la "bombe au radium " n'est efficace que si de grosses quantités, 4 ou 5 grammes de produits radioactifs, sont utilisées. Or, à 1,5 million de francs-or le gramme, le prix d'une seule bombe au radium correspond au montant annuel des subventions publiques envisagées pour le budget d'équipement des centres. D'où l'idée avancée par Regaud dans son rapport de limiter à une poignée le nombre de centres anticancéreux et d'en faire des centres de soins exemplaires, dotés des moyens suffisants [7]. La victoire politique de cette conception marquera profondément l'évolution de la cancérologie française, sanctionnant l'idée d'une médecine scientifique fondée sur l'utilisation d'une technologie lourde. Elle aura pour conséquences : (1) la construction de cinq centres "pilotes" équipés de bombes au radium et/ou d'appareils de roentgenthérapie ultrapénétrante constitués de tubes géants reliés à des générateurs de 800000 volts; (2) le 
développement d'un champ institutionnel profondément différencié puisque, en dehors de ces cinq centres, le traitement du cancer ne bénéficiera pas des innovations technologiques apparues pendant l'entredeux-guerres ; (3) l'affirmation du rôle incontournable de l'État dans l'équipement anticancéreux. Ce dernier point est crucial, car il signifie que le secteur libéral en France est incapable d'assumer le financement des technologies de pointe. Or, ce secteur assure en principe seul la prise en charge des malades payants, c'est-à-dire des classes moyennes et supérieures, tandis que le secteur public, lui, accueille les indigents. D'où cette situation paradoxale qui aboutit au fait que les "riches" ne bénéficient pas des progrès techniques. On comprend alors pourquoi les questions posées par le développement de la radiothérapie vont se retrouver au cœur d'un débat sur la "nécessaire réforme des hôpitaux", débat qui aboutira, après la Seconde Guerre mondiale, à la redéfinition de l'hôpital public comme "temple de la médecine moderne " ouvert à toutes les classes sociales, donc concurrentiel par rapport au secteur libéral [5]

\section{TIRÉS À PART}

P. Pinell.

\section{RÉFÉRENCES}

1. Bergonié J. Où en sont aujourd'hui les applications a la médecine et a la chirurgie de la découverte de Roentgen? J Med Bordeaux $1897 ; 27: 14-22$.

2. Lachapelle AP. La radiothérapie en 1905. J Radiol Electrol $1958 ; 39: 383-96$.

3. Bergonié J, Tribondeau L. Action des rayons X sur le testicule. Arch Elec Med $1906: 1-52$.

4. Jamous H. La réforme des éludes médicales el des structures hospitalieres. Paris : (inrs, centre d'études sociologiques, 1987.

5. Pinell P. Naissance d'un Réau. Histoire de la lutte contre le cancer en France (189()-1940). Paris : A.M. Métailié, 1992.

6. Bergonié J. Comment doivent être organisés les centres régionaux de lutte contre le cancer. Paris Médical 1923; 48: 146-9.

7. Regaud C. Doit-on augmenter le nombre de centres de thérapeutique anticancéreuse ? Rapport fait à la Commission du cancer. La lutte contre le Cancer 192:) ; 10 : 13744 .

\section{Patrice Pinell}

Directeur de l'unité de recherches Inserm U. 158, université René-Descartes - Paris $V$, hôpital des Enfants Malades, pavillon Archambault, 149, rue de Sèures, 75015 Paris, France.

\begin{tabular}{|c|c|}
\hline $\begin{array}{r}\text { Groupe de réflexion } \\
\text { sur la }\end{array}$ & $\begin{array}{c}\text { BIOLOGIE } \\
\text { ET PATHOLOGIE } \\
\text { DU COEUR } \\
\text { ET DES VAISSEAUX }\end{array}$ \\
\hline \multicolumn{2}{|c|}{$\begin{array}{c}\text { LYON, } 2 \text { et } 3 \text { avril } 1996 \\
\text { PALAIS des CONGRÉS } \\
\text { Cité Internationale } \\
\text { uai Charles-de Gaulle - } 69006 \text { Lyon - France }\end{array}$} \\
\hline $\begin{array}{l}\text { Secrétariat scientifique : Docteur Michel Ovize } \\
\text { Service du Professeur Xavier André-Fouet } \\
\text { Hôpital Cardiologique et Pneumologique } \\
\text { Louis-Pradel, } \\
\text { 59, boulevard Pinel, } 69394 \text { Lyon Cedex } 03 \text {, France } \\
\text { Tél. : (33) } 72.35 .75 .90 \text { ou }(333) 72.35 .75 .92 \\
\text { Télécopie : (33) } 72.35 .73 .76\end{array}$ & $\begin{array}{l}\text { Agence congrès : Chairman } \\
\text { Les Portes d'Antigone, } \\
\text { 43, place Vauban, 34000 Montpellier, France } \\
\text { Téléphone : (33) } 67.15 .99 .00 \\
\text { Télécopie : (33) } 67.15 .99 .09\end{array}$ \\
\hline
\end{tabular}

